\title{
Underoil Superhydrophilic Metal Felt Fabricated by Modifying Ultrathin Fumed Silica Coatings for Separation of Water-in-Oil Emulsions
}

\author{
Chaolang Chen, ${ }^{\mathrm{a}, \mathrm{c}}$ Shuai Chen, ${ }^{\mathrm{b}}$ Lei Chen, ${ }^{\mathrm{a}}$ Yadong Yu, ${ }^{\mathrm{a}}$ Ding Weng, ${ }^{\mathrm{a}}$ Awais Mahmood, ${ }^{\mathrm{a}}$ \\ Jiadao Wang, ${ }^{* a}$ Ivan P. Parkin ${ }^{\mathrm{c}}$, Claire J. Carmalt ${ }^{\mathrm{c}}$ \\ a Sate Key Laboratory of Tribology, Tsinghua University, Beijing 100084, P.R. China \\ b Institute of High Performance Computing, A*STAR, 138632, Singapore \\ c Department of Chemistry, University of College London, 20 Gordon Street, London \\ WC1H 0AJ, UK
}

\begin{abstract}
Although various superhydrophobic/superoleophilic porous materials have been developed and successfully applied to separate water-in-oil emulsions through sizesieving mechanism, the separation performance is restricted by their nanoscale pore size severely. In this study, the wettability of underoil water on fumed silica was experimentally observed and underlying mechanism was investigated by carrying out theoretical analysis and molecular dynamic (MD) simulations. Further, we present a novel, facile and inexpensive technique to fabricate underoil superhydrophilic metal felt with micro pores for separation of water-in-oil emulsions by using $\mathrm{SiO}_{2}$ nanoparticles (NPs) as building blocks. The as-prepared underoil superhydrophilic coating is closed-packed and ultrathin (the thickness is approximate hundreds of nanometers), as well as capable of being coated on metal felt with complex structures
\end{abstract}


without blocking its pores. The as-prepared metal felt could adsorb water droplets directly from oil, which endowed it with the ability to separate both surfactant-free and surfactant-stabilized water-in-oil emulsions with high separation efficiency up to $99.7 \%$ even though its pore size is larger than that of the emulsified droplet. The filtration flux for separation of span 80 -stabilized emulsion is up to $\sim 4000 \mathrm{~L} \cdot \mathrm{m}^{-2} \cdot \mathrm{h}^{-1}$. Its separation performance is better than most of the other traditional membranes and superwettable materials used for separation of water-in-oil emulsions. Moreover, the as-prepared metal felt retained outstanding separation performance even after 30 cycles of use, which demonstrated its excellent reusability and durability. Additionally, the distinctive wettability of underoil superhydrophilicity endued coated metal felt with superior anti-fouling properties towards crude oil. Overall, this study not only provides a new perspective on separating water-in-oil emulsion, but also gives a universal approach to develop special wettability materials.

Key words: underoil superhydrophilic; fumed silica; nanoparticle; water-in-oil emulsion; oil water separation.

\section{INTRODUCTION}

Oil-water mixtures generated from daily life and industrial production have caused not only serious environmental pollution, but also the waste of treasured resources. ${ }^{1-3}$ Moreover, tiny amounts of water in fuel can condense on the metal surface of an engine to produce corrosion, which greatly shorten its service-lifetime and increase the risk of engine failure. ${ }^{4-6}$ Therefore, separation of oil/water mixtures has drawn growing 
interests of scientists and engineers all over the world. Conventional treatment techniques including gravity separation, centrifuge, air-flotation and biodegradation, have been employed to separate stratified oil/water mixtures. ${ }^{7-9}$ Nevertheless, the emulsified droplets in an emulsion usually have a diameter less than $20 \mu \mathrm{m}$ and are extremely stable due to the adsorption of surfactant at the oil/water interface. ${ }^{10-12}$ They do not easily coalesce and cannot easily be separated by aforementioned traditional methods.

Recently, porous superwetting materials with pore size smaller than that of emulsified droplets in the emulsion have been developed to achieve the separation of emulsified oil/water mixtures by intercepting emulsified droplets. ${ }^{5}$ Generally, these materials are divided into two types: oil-blocking and water-blocking materials. The oil-blocking materials with superhydrophilic and underwater superoleophobic properties can allow the water to pass through a membrane while repelling the oil completely. The water-blocking materials with superoleophilic and underoil superhydrophobic properties can selectively filter oil from an emulsion. So far, diverse oil-blocking and water-blocking materials have been widely developed to separate various emulsions. ${ }^{13-16}$ For example, oil-blocking materials such as hydrogel-networks coated membranes, ${ }^{17} \mathrm{TiO}_{2}$ glass fibrous membrane, ${ }^{18}$ zinc oxide coated membranes ${ }^{19}$ and so forth, ${ }^{20,21}$ have been widely applied to separate oil-in-water emulsions. Waterblocking materials such as PPS microporous membranes, ${ }^{22}$ superhydrophobic 1H,1H,2H,2H-Perfluuorodecyltriethoxysilane-paper@ZnO paper, $^{23}$ polydopamine/silver NPs modified poly(lactic acid) membranes ${ }^{24}$ and so forth, ${ }^{25,} 26$ 
have also been commonly employed for the separation of water-in-oil emulsions. Considering the size distribution of emulsified droplets usually ranges from hundreds of nanometers to tens of micrometers, these materials normally possess nanoscale pore size in order to achieve effective interception of emulsified water droplets in water-inoil emulsions. ${ }^{27}$ These separation materials based on "size-sieving" effect greatly lower the separation flux and limit the separation efficiency, as well as increases energy consumption. Furthermore, their pores tend to be plugged by the accumulated emulsified droplets, which results in a sharp decline of flux within a few minutes. In addition, they are easily contaminated by oil due to their high affinity towards oil. These drawbacks limit their application severely in practical industry. ${ }^{28-31}$ Therefore, it is particularly needed to develop novel oil/water separators that do not rely on size-sieving separation.

Very recently, it was found that underoil superhydrophilic materials that exhibit super-amphiphilic property in air and superhydrophilicity in oil could adsorb emulsified water droplets directly from water-in-oil emulsion. ${ }^{32,33}$ During separation process, the emulsified water droplets would wet, coalesce and demulsify once contact the surface of underoil superhydrophilic materials. Therefore, separator with underoil superhydrophilic property can maintain efficient separation without greatly reducing its pore size. Although underoil superhydrophilic materials possess unique advantages over "water-blocking" superwetting materials in terms of separation performance and anti-fouling properties, it is still difficult to fabricate underoil superhydrophilic surfaces due to the fact that the oil phase tends to be trapped into the amphiphilic nano/micro 
structures and results in the enhancement of water-repellency. ${ }^{34}$ Lately, we accidentally found that fumed silica exhibits a unique underoil superhydrophilic property, which make it a promising candidate of developing underoil superhydrophilic surfaces. In this study, the wettability of underoil water on fumed silica was experimentally observed and underlying mechanism was investigated by carrying out theoretical analysis and MD simulations. To our knowledge, this is the first report on the underoil wettability of silicon dioxide.

Further, we presented a simple, universal and cost-efficient method to fabricate underoil superhydrophilic metal felt based on $\mathrm{SiO}_{2}$ NPs. The as-prepared $\mathrm{SiO}_{2}$ coating is ultrathin and exhibits uniform coverage. The as-prepared metal felts show both superamphiphilicity in air and superhydrophilicity in oil, as well as excellent antifouling properties towards crude oil. Besides, the as-prepared metal felts could adsorb water droplets from oil and achieve rapid and highly efficient separation of diverse surfactant-free and surfactant-stabilized water-in-oil emulsions under a solely gravity driver. Additionally, the as-prepared metal felts exhibited superior recyclability and outstanding long-term stability.

\section{METHODS}

\section{Experimental Materials}

Stainless-steel metal felts were available commercially. EVO-STIK Serious Glue was obtained from Bostik Co. Ltd. Fumed silica having an average diameter of $16 \mathrm{~nm}$ was purchased from Degussa, Germany. Diesel was obtained from a local garage. Crude oil 
was obtained from Sinopec Group Co. Ltd, China. All other chemical reagents including oils and organic solvents used in this work were of analytical grade. All the aqueous solutions were prepared using deionized water.

\section{Preparation of underoil superhydrophilic coating}

Firstly, the metal felt was ultrasonically washed by orderly immersed in acetone, ethanol and deionized water for 10 minutes to remove contaminants. $1 \mathrm{~g}$ of EVO-STIK Serious Glue was added into $99 \mathrm{~g}$ of ethanol and the solution was electromagnetically stirred for 30 minutes to obtain a diluted glue solution. $4 \mathrm{~g}$ of fumed silica was placed into $96 \mathrm{~g}$ of deionized water and the solution was mechanically stirred for 2 hours and ultrasonically dispersed for 10 minutes to obtain a $\mathrm{SiO}_{2}$ colloidal suspension. Secondly, the cleaned metal felt was dipped in diluted glue solution for 10 minutes and then dried at $80{ }^{\circ} \mathrm{C}$ to obtain glue modified metal felt. Subsequently, the glue modified metal felt was immersed into $\mathrm{SiO}_{2}$ colloidal suspension for 10 minutes, then rinsed by deionized water and dried at $80{ }^{\circ} \mathrm{C}$. Finally, the coated metal felt was annealed at $550{ }^{\circ} \mathrm{C}$ for 2 hours to remove the glue and immobilize the $\mathrm{SiO}_{2}$ NPs onto the felt surface.

\section{Water-in-oil emulsion separation experiment}

In order to test the separation performance of the as-prepared metal felts, various surfactant-free and surfactant-stabilized water-in-oil emulsions were prepared. $1 \mathrm{~g}$ of water was added into $99 \mathrm{~g}$ of various oils (diesel, petroleum ether, decane and Ntetradecane) and then ultrasonically stirred for 5 minutes to prepare surfactant-free 
water-in-oil emulsions. 0.02 wt.\% of Span 80 was added into surfactant-free water-inoil emulsions so as to a prepare surfactant-stabilized water-in-oil emulsion. A homemade device was applied during oil/water separation tests in which the asprepared metal felts were fixed at the end of a glass tube. A silicone-sealing ring was installed between the felt and the glass tube in order to guarantee the stability of the seal. The separation efficiency was calculated by the following equation (1):

$$
\eta=\frac{C_{0}-C_{1}}{C_{0}} \times 100 \%
$$

Where $\eta$ is the separation efficiency, $C_{0}$ is the water content in original water-inoil emulsions (ppm) and $C_{1}$ is the water content in the collected filtrate (ppm).

\section{Characterizations}

The surface topography and chemical components were examined by scanning electron microscope (SU8220, HITACHI) and energy dispersive spectroscopy (EDS, Bruker). The pore size distributions and porosity of raw and coated metal felt were measured by means of a Mercury Porosimeter ( Autopore IV 9510, Micromeritics Instruments Corporation, USA). The surface wettability property of pristine and coated metal felt was evaluated by measuring their liquid contact angle via OCA 25 (Data-Physics, Germany; water or oil droplet with volume of $4 \mu \mathrm{L}$ were used). The water content in collected filtrates was measured by a Karl Fischer Titrator (TP653, TimePower Measure and Control Equipment Co. Ltd). The size dispersion of water droplets in oil was measured using a Zetasizer Nano ZS (Malvern). The microscope images of water-inoil emulsions were recorded using an inverted microscope. 


\section{Molecular dynamics simulations}

For the simulations, a model composed of water on oil-wetted $\mathrm{SiO}_{2}$ was constructed as shown in Figure S1. The lateral directions of the model were periodic with dimensions of $L_{x}=L_{y}=6 \mathrm{~nm}$. The vertical direction of the model was non-periodic and the thickness of $\mathrm{SiO}_{2}$, oil (hexane $\mathrm{C}_{6} \mathrm{H}_{14}$ was used as an example) and water were $2 \mathrm{~nm}, 1 \mathrm{~nm}$ and 1 $\mathrm{nm}$, respectively. Quartz (100) surface was used to model $\mathrm{SiO}_{2}$ with hydroxyl (-OH) or hydro $(-\mathrm{H})$ groups as a fixed substrate, whose parameters were taken from Lopes et al.'s research ${ }^{35}$. The water model used the rigid extended simple point charge potential $(\mathrm{SPC} / \mathrm{E})^{36}$. The oil model is $\mathrm{C}_{6} \mathrm{H}_{14}$ molecule from Moultos et al.'s research ${ }^{37}$, which was also kept rigid during the simulation. The electrostatic interaction was modeled using Coulomb's law, while the dispersion and repulsion forces were based on the Lennard-Jones $(\mathrm{L}-\mathrm{J})$ potential. More details on the geometrical and force field parameters are described in supporting information Table S1 to S3. All simulations were performed in the NVT (fixed number of particles, volume, and temperature) ensemble at a temperature of $300 \mathrm{~K}$ by LAMMPS ${ }^{38}$. The simulation time was $10 \mathrm{~ns}$ with an integration time step of $1 \mathrm{fs}$, and the samples of the trajectories were stored every $0.1 \mathrm{~ns}$. 


\section{Results and Discussion}

\section{Underoil wettability of $\mathrm{SiO}_{2} \mathrm{NP}$ plate}

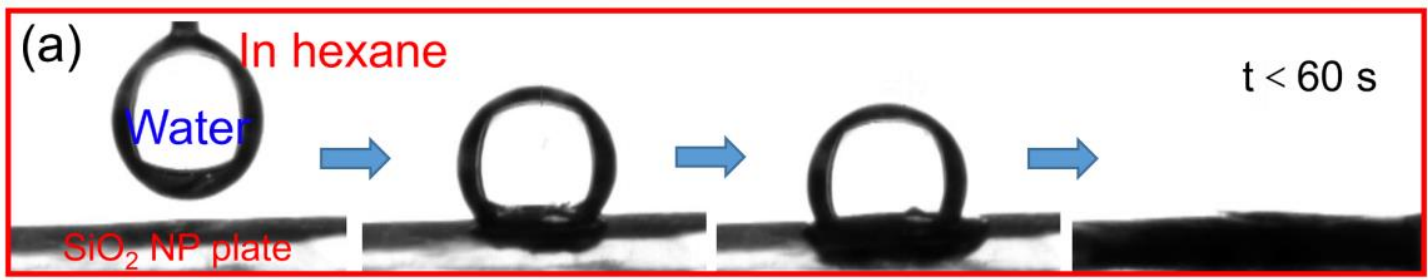

(b) In hexane

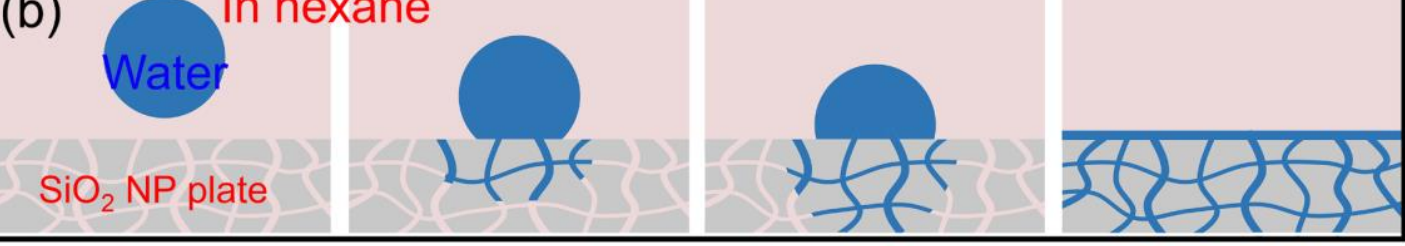

Figure 1. (a) Experimental snapshots and (b) corresponding illustration of a water droplet spread on the $\mathrm{SiO}_{2} \mathrm{NP}$ plate that was immersed in hexane environment.

The wettability of underoil water on silicon dioxide was experimentally observed and underlying mechanism was investigated by carrying out theoretical analysis and MD simulations. Pure $\mathrm{SiO}_{2}$ NP plates were prepared through a Tablet Press Machine (experimental parameters: $0.1 \mathrm{~g}$ of fumed silica; $5 \mathrm{KN}$ of load; $30 \mathrm{~s}$ of holding time). As displayed in Figure S2, the as-prepared $\mathrm{SiO}_{2} \mathrm{NP}$ plate was consisted of abundant $\mathrm{SiO}_{2}$ NPs and exhibited a smooth surface appearance at micro level. The wetting process of an underoil water droplet on $\mathrm{SiO}_{2} \mathrm{NP}$ plate was recorded as shown in Figure 1a and Movie S1. It can be observed that the water droplet was fully adsorbed by the plate within $60 \mathrm{~s}$, exhibiting an underoil superhydrophilic feature. As illustrated in Figure $1 \mathrm{~b}$, the $\mathrm{SiO}_{2}$ NP plate surface was completely wetted by oil (hexane as an example) and the gaps between $\mathrm{SiO}_{2}$ NPs were filled with oil when being immersed in oil. However, during the process of water contacting $\mathrm{SiO}_{2} \mathrm{NP}$ plate, these oils on $\mathrm{SiO}_{2}$ 
surface were replaced by water gradually until realizing a thermodynamic equilibrium state.

When a water droplet stays stably on an ideal smooth solid surface that was immersed in an oil environment, a thermodynamic equilibrium would be achieved among oil/water/solid three-phase interface as shown in Figure S3. As described by Young's equation:

$$
\cos \theta_{w o}=\frac{\gamma_{s o}-\gamma_{s w}}{\gamma_{w o}}
$$

Where $\gamma_{s o}$ is the interfacial energy at solid/oil interface, $\gamma_{s w}$ is the interface tension at solid/water interface; $\gamma_{w o}$ is the interface tension at water/oil interface. $\theta_{w o}$ is the underoil water contact angle on ideal flat solid surface.

For an underoil superhydrophilic surface, $\theta_{w o} \leq 90^{\circ}$. Therefore, $\gamma_{s o}$ must be larger than $\gamma_{s w}$, which means the solid surface has stronger affinity to water than oil, thus the oil would be replaced by water automatically.

To further reveal the underlying mechanism of the strong affinity between $\mathrm{SiO}_{2}$ and water, we performed MD simulations with water on oil (hexane)-wetted $\mathrm{SiO}_{2}$. Figure 2a to c show the snapshots in the wetting process of water on oil-wetted $\mathrm{SiO}_{2}$ with hydroxyl groups (-OH) (The dynamic wetting process is shown in Movie S2). The results indicate that water molecules first penetrate into the oil layer, subsequently start to wet the $\mathrm{SiO}_{2}$ surface and finally fully replace oil, which agree well with our experimental observations. When the hydroxyl groups $(-\mathrm{OH})$ of $\mathrm{SiO}_{2}$ are replaced with hydro groups $(-\mathrm{H})$, snapshots in the wetting process of water on oil-wetted $\mathrm{SiO}_{2}$ are drawn in Figure $2 \mathrm{~d}$ and e. It demonstrates that the water molecules can only partially 
replace oil when the functional groups of $\mathrm{SiO}_{2}$ are changed. Therefore, it can be concluded that the strong affinity between $\mathrm{SiO}_{2}$ and water should be attributed to the high surface energy of $\mathrm{SiO}_{2}$ and the abundant surface polar group $(-\mathrm{OH})$.

To quantitatively analyze the wetting process of water on $\mathrm{SiO}_{2}$ to replace oil, the ratio of adsorbed water and oil molecules (within $3 \AA$ distance from $\mathrm{SiO}_{2}$ top surface) during wetting process are recorded in Figure $2 \mathrm{f}$. Red and blue continuous lines represent the ratio of water and oil on $\mathrm{SiO}_{2}$ with hydroxyl groups, respectively. It indicates that water molecules wet $\mathrm{SiO}_{2}$ to replace oil dramatically in $\sim 3 \mathrm{~ns}$ and then occupy $\sim 80 \%$ of the $\mathrm{SiO}_{2}$ surface. After that, the replacement of water with oil slows down and gradually realizes $100 \%$ in $\sim 9$ ns. However, when the hydroxyl groups (-OH) of $\mathrm{SiO}_{2}$ are replaced with hydro groups $(-\mathrm{H})$, the ratio of adsorbed water molecules can only go up to $\sim 40 \%$ in $10 \mathrm{~ns}$ (red dashed line) and there still exists $\sim 60 \%$ of $\mathrm{SiO}_{2}$ surfaces that are wetted by oil, corresponding to the snapshot in Figure 2e. Therefore, the strong affinity between $\mathrm{SiO}_{2}$ coated metal felt and water could be attributed to the high surface energy of $\mathrm{SiO}_{2}$ and the abundant surface hydroxyl groups (-OH). Moreover, when a water droplet contacted the coated metal felt that was steeped in oil, the hydroxyl groups help the water to further wet on the surface because of the formation of hydrogen bonds. 

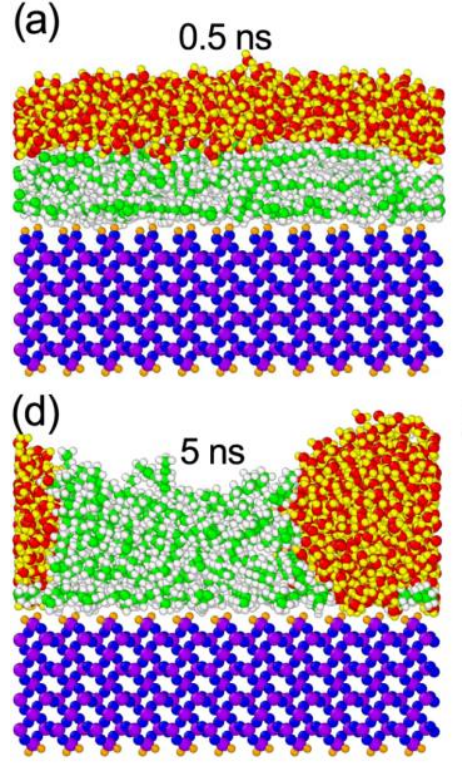
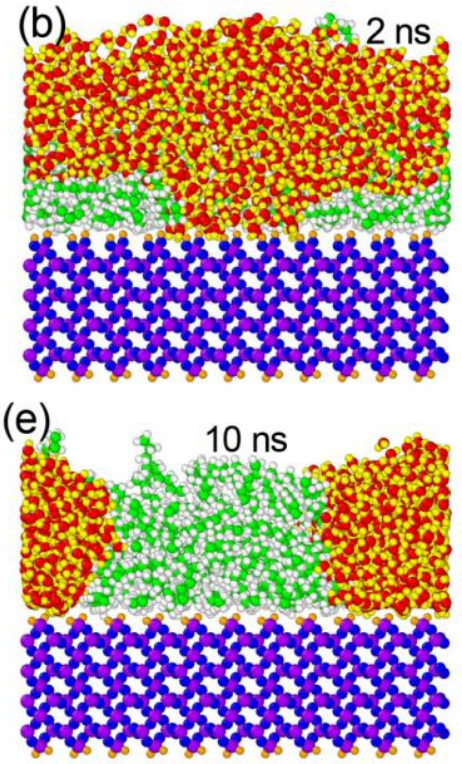
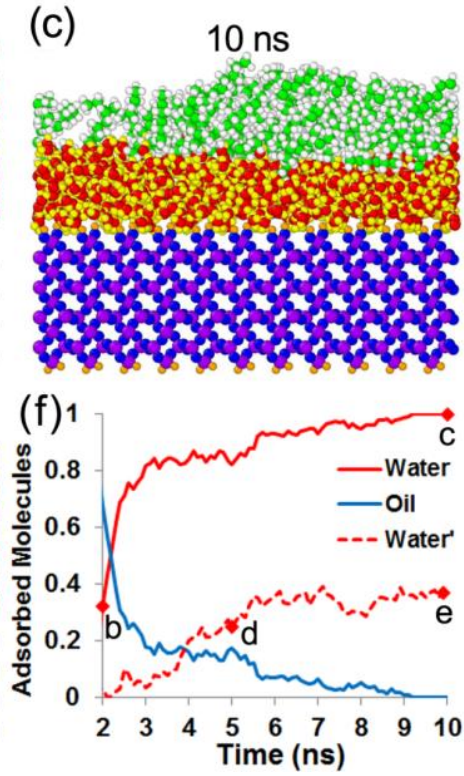

Figure 2. (a-c) Snapshots in the wetting process of water on oil-wetted $\mathrm{SiO}_{2}$ with hydroxyl groups $(-\mathrm{OH}) .(\mathrm{d}, \mathrm{e})$ Snapshots in the wetting process of water on oil-wetted $\mathrm{SiO}_{2}$ with hydro groups (-H). Blue, purple and orange spheres represent the $\mathrm{Si}$, O and $\mathrm{H}$ atoms of $\mathrm{SiO}_{2}$, respectively. Green and white spheres represent the $\mathrm{C}$ and $\mathrm{H}$ atoms of oil, respectively. Red and yellow spheres represent the $\mathrm{O}$ and $\mathrm{H}$ atoms of water, respectively. (f) Ratio of adsorbed water and oil molecules during wetting process. Red and blue continuous lines represent the ratio of water and oil on $\mathrm{SiO}_{2}$ with hydroxyl groups, respectively. Red dashed line represents the ratio of water on $\mathrm{SiO}_{2}$ with hydro groups. 


\section{Surface morphology and chemical component of $\mathrm{SiO}_{2}$ NP coated metal}

\section{felt}

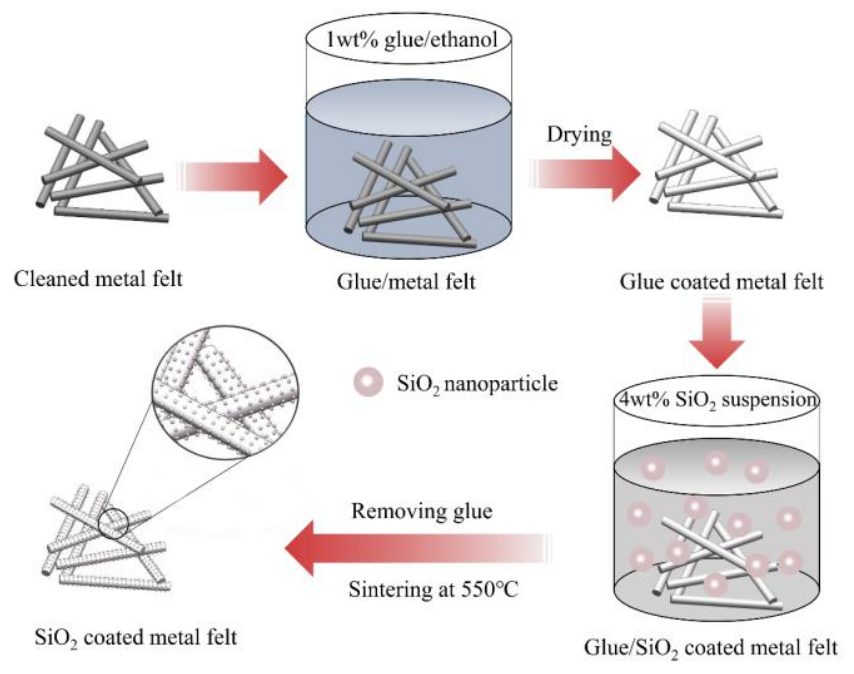

Figure 3. Schematic diagram of fabrication process for $\mathrm{SiO}_{2} \mathrm{NPs}$ modified metal felt

As illustrated in Figure 3, we fabricated glue and $\mathrm{SiO}_{2} \mathrm{NPs}$ coated metal felt though a two-step strategy of dipping in diluted glue solution and NP suspension, respectively, which has been demonstrated to be a facile method of uniformly modifying NP coatings onto 3D materials. ${ }^{39}$ The glue was used to adsorb NPs onto the metal fibers and form uniform coatings. After that, the glue was removed by heating treatment because it contains hydrophobic compounds that would reduce the affinity of as-prepared coating toward water. Meanwhile, annealing treatment could enhance the bonding between NP coating and substrate. ${ }^{40,41}$ As shown in Figure 4a, it can be seen that the raw metal felt is made up of nonwoven micro metal fibers with an average diameter of $7.91 \mu \mathrm{m}$. The high-resolution SEM images indicate that the metal fibers have a neat and smooth surface (Figure 4(b, c)). After coated with $\mathrm{SiO}_{2} \mathrm{NPs}$, the obtained metal fibers were fully wrapped by a dense $\mathrm{SiO}_{2}$ coating, and exhibited 
hierarchical micro-/nano-structures as shown in Figure 4(d-f). The diameter of metal fiber was counted based on its SEM images and using Nano Measure Software. The coated metal fibers displayed an average diameter of $8.49 \mu \mathrm{m}$, indicating the thickness of formed coating was at the submicron scale (coating thickness is $\sim 290 \mathrm{~nm}$ ). Furthermore, the pore size distributions and porosity of raw and coated metal felt were measured by means of Mercury Porosimeter as shown in Figure S4. The results show that the raw and coated metal felt exhibited similar pore distributions with average equivalent pore diameter of $\sim 11 \mu \mathrm{m}$. After coated with the $\mathrm{SiO}_{2}$ coating, the porosity of metal felt slightly decreased from $71 \%$ to $65.3 \%$, which indicated that the formed coating was ultrathin and the $\mathrm{SiO}_{2}$ NPs did not block the pores of the metal felt. EDS measurements were carried out to examine the chemical component of raw and coated metal felt. As shown in Figure 4(g, h), the results demonstrated that the main components of raw metal felt are $\mathrm{Fe}, \mathrm{Cr}$ and $\mathrm{Ni}$ elements, which is consistent with previously reported publications. ${ }^{8}$ Extra $\mathrm{Si}$ and $\mathrm{O}$ elements were detected in the coated metal felt and exhibited an atomic ratio of $\sim 1: 2$, which confirmed the coating is composed of silicon dioxide. As shown in Figure S5, the FTIR spectroscopy reveals that plenty of hydroxyl appeared on the metal felt after being coated with $\mathrm{SiO}_{2} \mathrm{NPs}$. In addition, the distribution of Si element in coated metal felt further confirmed that the as-prepared $\mathrm{SiO}_{2}$ coating shows excellent uniformity and uniform coverage (Figure 4i). 

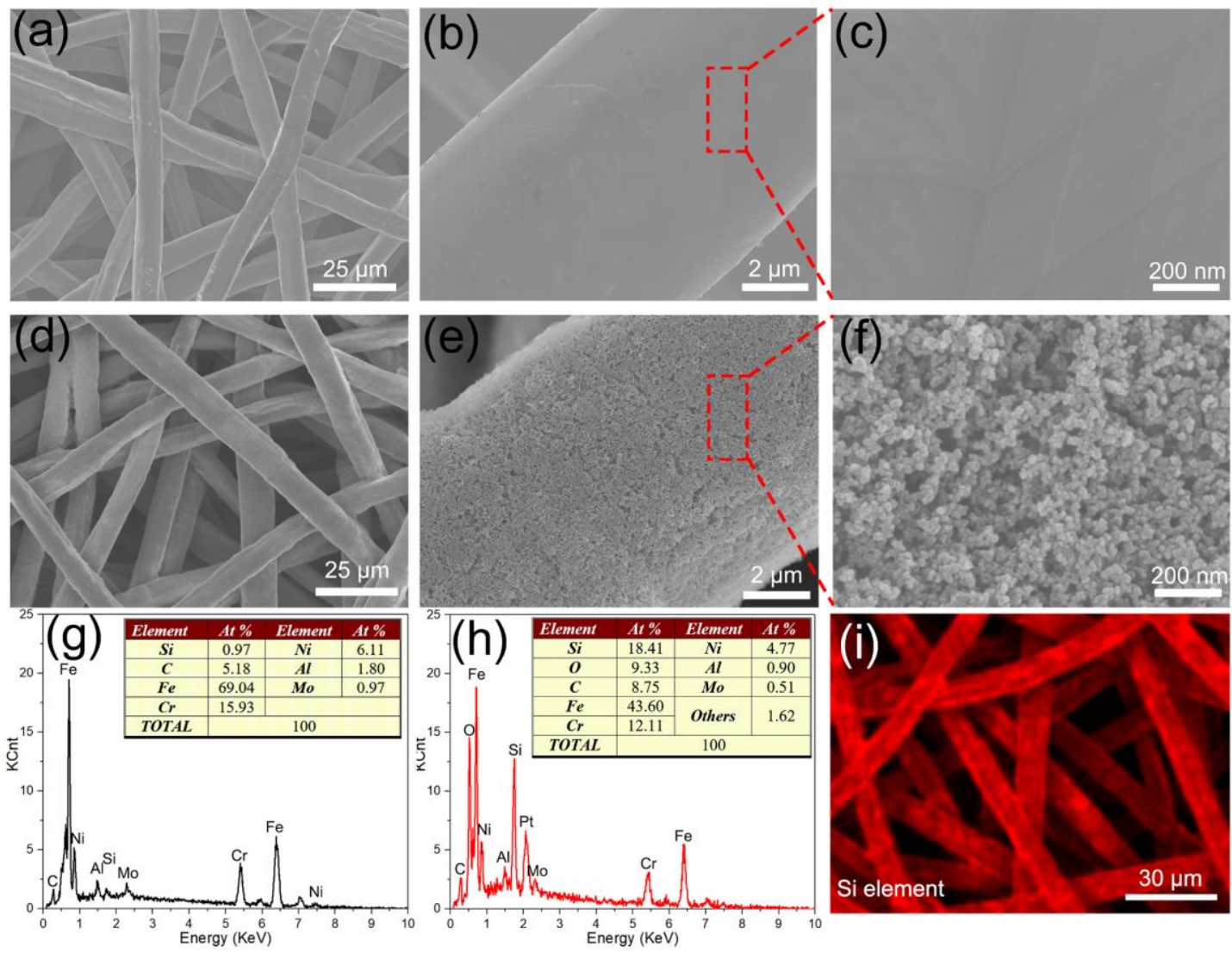

Figure 4. (a-c) SEM images of raw metal felt; (d-f) SEM images of $\mathrm{SiO}_{2}$ coated metal felt; (g) EDS spectrum of raw metal felt; (h) EDS spectrum and (i) the Si element mapping of as-prepared metal felt.

\section{Wettability of $\mathrm{SiO}_{2} \mathrm{NP}$ coated metal felt}

As shown in Figure S6a, the pristine metal felt was hydrophobic with a static water contact angle of $\sim 125^{\circ}$. After annealing treatment, the metal felt was oxidized and became superhydrophilic (Figure S6b). It can be seen that the glue exhibited hydrophobic property, which has to be removed in order to improve the affinity of coating towards water (Figure S6(c, d)). After modification with $\mathrm{SiO}_{2} \mathrm{NPs}$, the metal felt transformed from hydrophobic to superhydrophilic and superoleophilic in atmosphere (Figure 5(a, b)). Moreover, as shown in Figure 5c, the as-prepared felt 
exhibited a superhydrophilic property in oil while the metal felts without $\mathrm{SiO}_{2}$ coating were hydrophobic in oil (Figure S6). A water droplet quickly spread on the as-prepared felt immersed in diverse oil within one second (Figure 5d, Figure S7 and Movie S3), demonstrating the excellent underoil superhydrophilicity of the $\mathrm{SiO}_{2}$ coated metal felt.

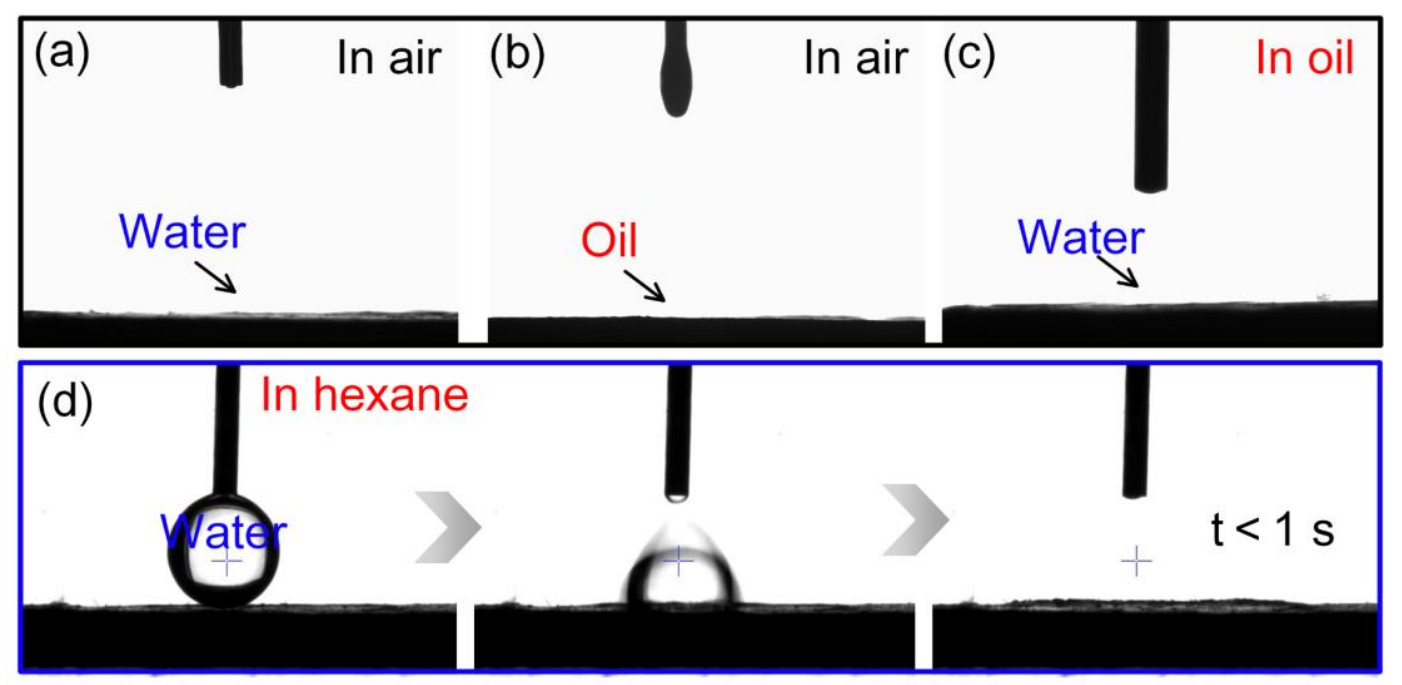

Figure 5. Wetting behavior of the as-prepared metal felt towards (a) water in air, (b) oil in air, (c) water in oil. (d) The snapshots of a water droplet wets and spreads on asprepared metal felt surface that was pre-immersed in oil phase.

\section{Separation of water-in-oil emulsions}

The unique underoil superhydrophilicity of $\mathrm{SiO}_{2}$ coated metal felt endowed it with the capability to capture water from an oil environment. As shown in Figure 6, a water droplet was placed at the bottom of a transparent box filled with oil. When the coated metal felt surface contacted the water droplet in oil, the water droplet spread out on the surface immediately and finally was fully removed from the oil (Movie S4). 


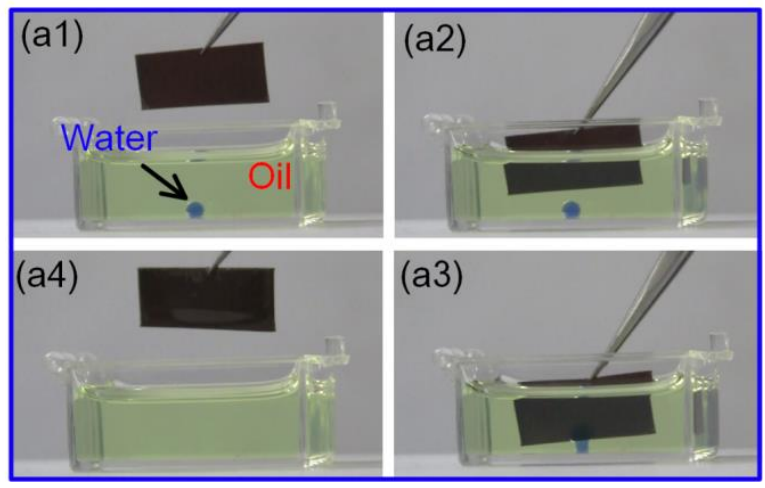

Figure 6. (a1-a4) The adsorption process of an underoil water (dyed with methyl blue) droplet from oil (diesel) by using as-prepared metal felt.

Further, the as-prepared metal felt with underoil superhydrophilic properties could separate diverse water-in-oil emulsions by adsorbing emulsified water from emulsions. In order to evaluate its separation performance, an experimental setup was built as shown in Figure 7a. During the separation process, emulsions were poured onto the asprepared metal felt to accomplish separation (as shown in Movie S5). Figure 7(b-d) shows the separation results of water-in-diesel emulsion. As displayed in Figure 7(b, c), the original water-in-diesel emulsion was extremely turbid and contained plenty of emulsified water droplets whose size distribution ranges from hundreds of nanometers to tens of micrometers. After permeating through the as-prepared metal felt, the collected filtrate become clear and transparent, and there was no emulsified water droplets could be observed by an optical microscope. Moreover, Figure $7 \mathrm{~d}$ reveals that only DLS signals at several nanometers could be detected in collected filtrate, which indicates the as-prepared metal felt with micro-scale pores could even "intercept" nanoscale-sized emulsified droplets, exhibiting excellent separation performance. 

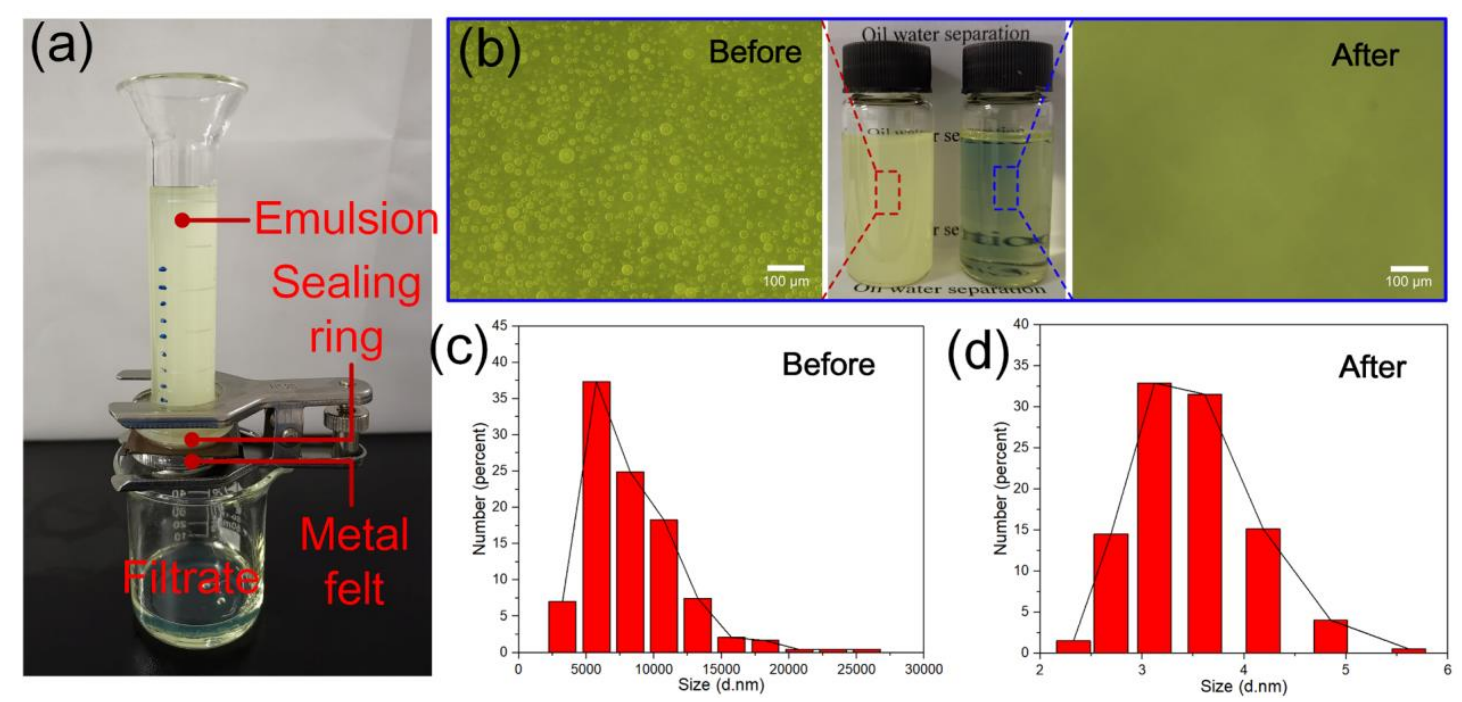

Figure 7. (a) Experimental setup for water-in-diesel emulsion separation. (b) The

digital images, optical microscope images, and water droplets size distribution of water-in-diesel emulsion (c) before and (d) after filtration.

The separation performance of as-prepared metal felt was further evaluated quantificationally by measuring the separation flux, water content in filtrate and calculating corresponding separation efficiency. As displayed in Figure 8a, the asprepared metal felt could effectively separate various surfactant-free water-in-oil emulsions including water-in-diesel, water-in-petroleum ether, water-in-decane and water-in-N-tetradecane with separation efficiency above $99.6 \%$ and the water content in filtrates less than $50 \mathrm{ppm}$. Furthermore, the as-prepared metal felt exhibited a high separation efficiency up to $99.4 \%$ for span 80 -stabilized water-in-oil emulsion (Figure 8b). Moreover, the filtration flux of as-prepared metal felt was calculated by the following equation: $L=V / S t$, where $V$ is the volume of collected filtrate, $S$ is the filtration area, $t$ is filtration time and the fluxes $(L)$ for diverse emulsions were calculated for one minute. Figure 8c shows that the separation fluxes for span 80stabilized water/diesel, water/petroleum, water/decane, and water/N-tetradecane 
emulsions were about $780 \mathrm{~L} \cdot \mathrm{m}^{-2} \cdot \mathrm{h}^{-1}, 3847 \mathrm{~L} \cdot \mathrm{m}^{-2} \cdot \mathrm{h}^{-1}, 1476 \mathrm{~L} \cdot \mathrm{m}^{-2} \cdot \mathrm{h}^{-1}$, $861 \mathrm{~L} \cdot \mathrm{m}^{-2} \cdot \mathrm{h}^{-1}$, respectively, which is ten times higher than that of the traditional membrane, as well as much larger than that of the other superwetting oil/water materials as summarized in Table 1. The reason of different types of water-in-oil emulsions exhibited very different flux can be explained by classic Hagen-Poiseuille theory as following: ${ }^{42} J=\varepsilon \pi r^{2} \Delta P / 8 \mu L$, where $J$ is filtration flux; $\varepsilon$ and $r$ represent the porosity and pore size of membrane, respectively; $\Delta P$ represents the pressure difference across membrane; $L$ represents the thickness of membrane and $\mu$ is the viscosity of liquid. As predicted by Hagen-Poiseuille theory, the separation flux of water-in-oil emulsions is inversely proportional to the viscosity of oil, which is consistent with the experimental results. Moreover, compared to the other filtration materials, fumed silica coated metal felt exhibited superior separation flux was mainly attributed to the feature that it possesses micro-scale pores.

Besides, the effect of the thickness of the filter on its separation performance was investigated by taking span 80 -stabilized water-in-diesel as an example. As exhibited in Figure 8d, it can be clearly seen that the separation efficiency of the emulsion gradually increased from $96.6 \%$ to $99.6 \%$ with the increase of thickness of the filter (from $0.44 \mathrm{~mm}$ (one layer of metal felt) to $2.2 \mathrm{~mm}$ (five layers of metal felt)), and the water content in collected filtrates decreased from $402 \mathrm{ppm}$ to $50 \mathrm{ppm}$. This is because that thicker filter has a larger effective separation area, which increased the chance of collision between emulsified water droplets and metal fibers. In addition, the separation flux of the emulsion decreased from $1733 \mathrm{~L} \cdot \mathrm{m}^{-2} \cdot \mathrm{h}^{-1}$ to $686 \mathrm{~L} \cdot \mathrm{m}^{-2} \cdot \mathrm{h}^{-1}$ (Figure 
S8), which is consistent with the prediction of Hagen-Poiseuille theory in which the filtration flux of porous material is inversely proportional to its filtration thickness.
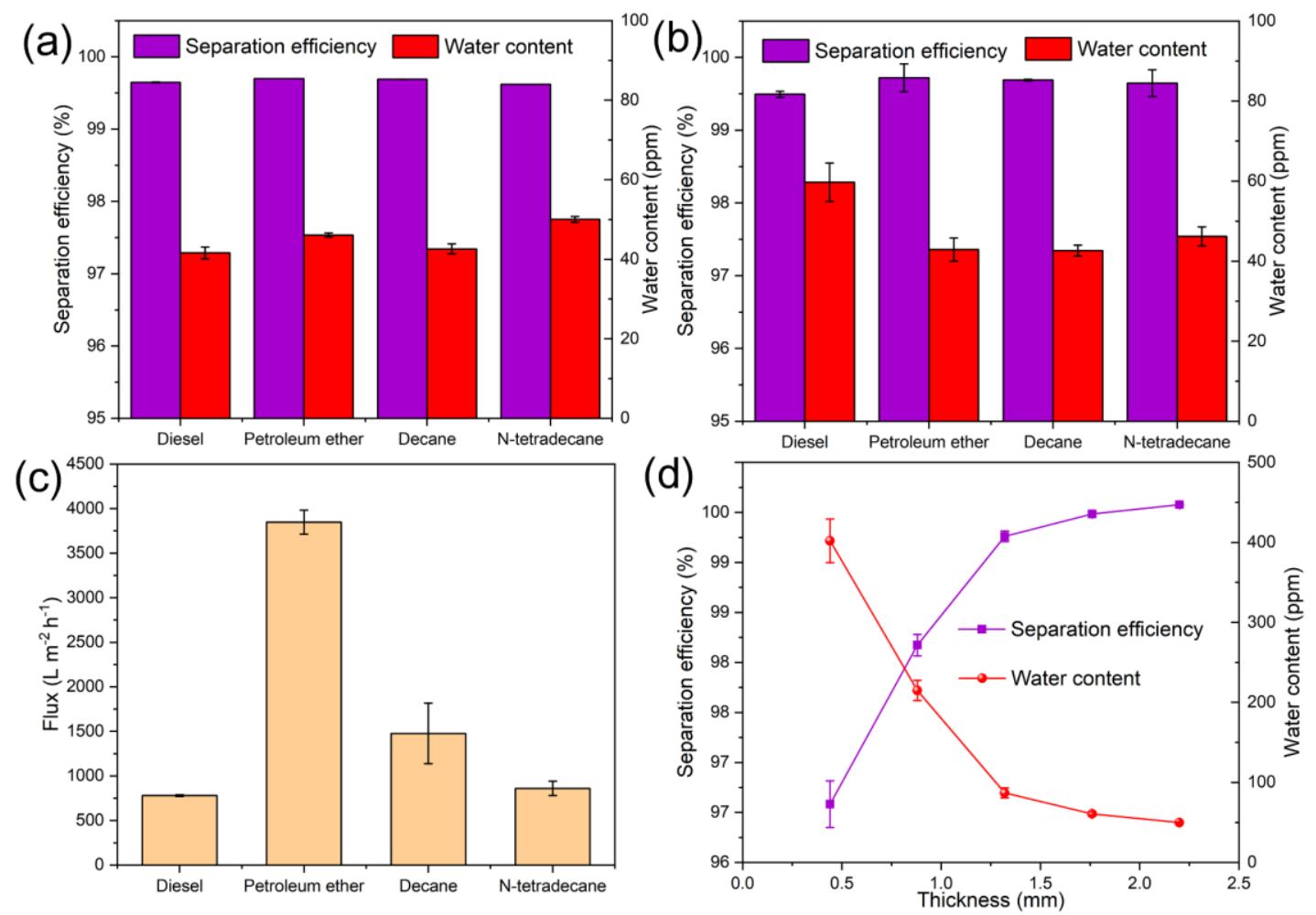

Figure 8. The separation efficiency and water content in the collected filtrates for (a) surfactant-free and (b) span 80-stabilized water-in-oil emulsions. (c) The separation fluxes of diverse span 80-stabilized water-in-oil emulsions. Experimental parameters: the thickness of filter is $1.76 \mathrm{~mm}$; the separation process is driven by solely gravity. (d) The relationship between the thickness of filter and its separation performance, span 80 -stabilized water-in-diesel was took as an example.

Table 1. Comparison of diverse filtration materials for separation of surfactant-stabilized water-inoil emulsion.

\begin{tabular}{lllll}
\hline Materials & Wettability & Driver & Flux & Ref \\
& & & \\
& & & \\
& & & \\
& & & \\
& & & \\
\end{tabular}




\begin{tabular}{|c|c|c|c|c|c|}
\hline $\mathrm{CNFs}^{1}-\mathrm{PDMS}^{2}$ & Superhydrophobic & Span 80-water-in- & Gravity & 984 & {$[43]$} \\
\hline inlay-gated $\mathrm{SSM}^{3}$ & /superoleophilic & toluene & & & \\
\hline \multirow[t]{2}{*}{ PVDF membrane } & Superhydrophobic & Span 80-water-in- & Gravity & $700-100$ & {$[12]$} \\
\hline & /superoleophilic & petroleum ether & & & \\
\hline $\mathrm{PVDB}^{4} / \mathrm{PVDF}$ & Superhydrophobic & Span 80-water-in- & $0.1 \mathrm{MPa}$ & $500-600$ & [44] \\
\hline Membrane & /superoleophilic & diesel & & & \\
\hline Co-PDMS@ZIF5 & Superhydrophobic & Span 80-water-in- & Gravity & 170 & {$[45]$} \\
\hline 8/MWCNT ${ }^{6}$ & /superoleophilic & toluene & & & \\
\hline Polypropylene & Superhydrophobic & Span 80 -water-in & $0.09 \mathrm{MPa}$ & 689 & {$[46]$} \\
\hline membrane & /superoleophilic & kerosene & & & \\
\hline \multirow[t]{2}{*}{ Desert sand } & Underoil & Span 80-water-in- & Gravity & 400 & {$[32]$} \\
\hline & superhydrophilic & diesel & & & \\
\hline \multirow[t]{2}{*}{$\mathrm{SiO}_{2} @$ metal felt } & Underoil & Span 80-water-in- & Gravity & 3847 & This \\
\hline & superhydrophilic & petroleum ether & & & work \\
\hline
\end{tabular}

Note: $\mathrm{CNFs}^{\mathrm{l}}=$ Carbon nanofibers; $\mathrm{PDMS}^{2}=$ Polydimethylsiloxane; $\mathrm{SSM}^{3}=$ Stainless steel mesh; $\mathrm{PVDB}^{4}=$ Polydivinylbenzene; $\mathrm{ZIF}^{5}=$ Zeolitic imidazolate framewor; $\mathrm{MWCNT}^{6}=$ Multiwalled carbon nanotubes.

The dynamic contacting process between emulsified droplets and as-prepared metal felt was observed by an inversed microscope as shown in Figure S9 (Movie S6). When the as-prepared metal felt was immersed in water-in-diesel emulsion, the emulsified droplets moved toward the metal felt and were immediately adsorbed by the fibers once contacted the metal felt's surface. Therefore, as illustrated in Figure 9a, the 
separation mechanism can be inferred as following: firstly, when contacting with waterin-oil emulsion, the as-prepared metal felt was fully wetted by the oil phase due to its superamphiphilic property in air, which could allow the oil phase permeate through freely. Secondly, the emulsified water droplets replaced oil molecules and spread on the metal fibers when moving toward the as-prepared metal felt. Finally, the adsorbed water film on fibers could further attract and coalesce with other emulsified droplets, thus achieving the efficient separation of water-in-oil emulsions. Moreover, a water film would form on metal felt with the increasing adsorption of emulsified water droplets, which would block oil phase from going through metal felt, meaning the metal felt has achieved a saturated state and cannot adsorb more emulsified droplets. However, the metal felt can continue to adsorb emulsified droplets after simple cleaning.

The reusable separation performance of as-prepared metal felt was evaluated by using span 80-stabilized water-in-diesel as an example. Each separation cycle lasted 10 minutes, and then the felt was ultrasonically washed by absolute ethanol for 5 minutes. After completely dried in oven at $80{ }^{\circ} \mathrm{C}$, the felt was used for the next separation cycle test. As exhibited in Figure 9b, it can be clearly observed that the separation efficiency always maintained higher than $99.4 \%$ and water content in filtrates always kept less than $80 \mathrm{ppm}$ even after 30 cycles of usage, which demonstrated the excellent reusability of as-prepared metal felt. Moreover, there is no obvious decline of filtration flux during the reusability measurement, which indicated the as-prepared metal felt exhibits splendid stability for filtration flux when separating water-in-oil emulsions (Figure 9c). 
(a) $\quad \mathrm{SiO}_{2}$ coated metal fiber
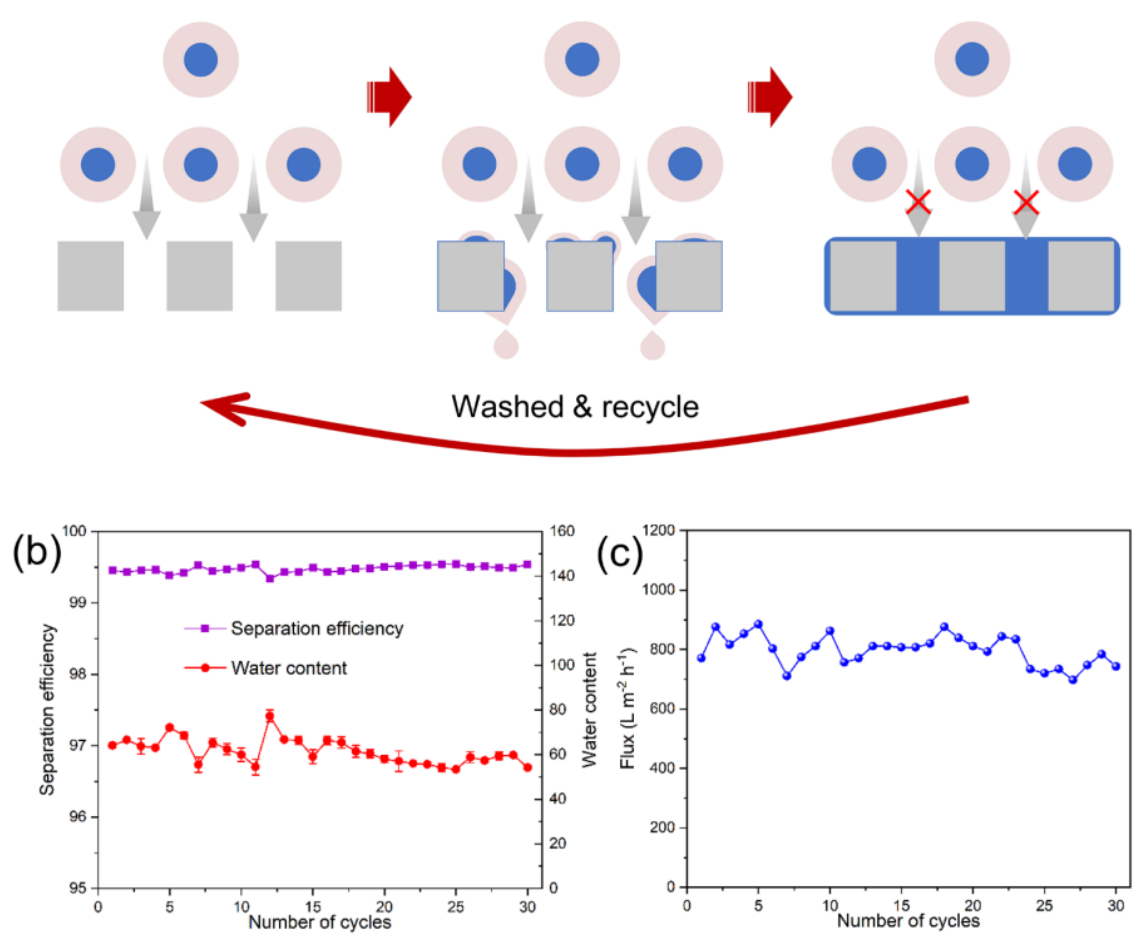

Figure 9. Reusable performance of as-prepared metal felt by taking span 80-stabilized water-in-diesel emulsion as an example. (a)Illustration of recycle of as-prepared metal

felt for separating water-in-oil emulsion. (b) Relationship between separation efficiency \& water content in filtrate and number of cycles. (c) The separation flux stability after 30 cycles of usage.

\section{Anti-fouling property}

A superhydrophilic surface with excellent water affinity is a promising candidate to prepare anti-fouling surfaces. As exhibited in Figure 10 (a-b), the as-prepared metal felt showed an underwater oil contact angle of $160^{\circ}$ and a small underwater oil slidingangle of $1^{\circ}$. Moreover, the oil could detach from the surface after serious deformation under external pressure (Figure 10c). The results demonstrated that the as-prepared 
metal felt exhibited excellent underwater superoleophobic property. Further, anti-crude oil fouling tests were carried out to evaluate the self-cleaning performance against crude oil of $\mathrm{SiO}_{2}$ modified metal felt. As shown in Figure 10d, a jet of crude oil could bounce off the felt surface that was immersed in water, without leaving any oil residual. When the $\mathrm{SiO}_{2}$ modified metal felt was dipped into crude oil/water mixture, the felt could maintain clean in water without any adhesion of floating crude oil (Figure 10e). It is truly believed that the unique underoil superhydrophilic property of $\mathrm{SiO}_{2}$ modified metal felt endows it with superior anti-fouling capability compared to other underwater superoleophobic materials.
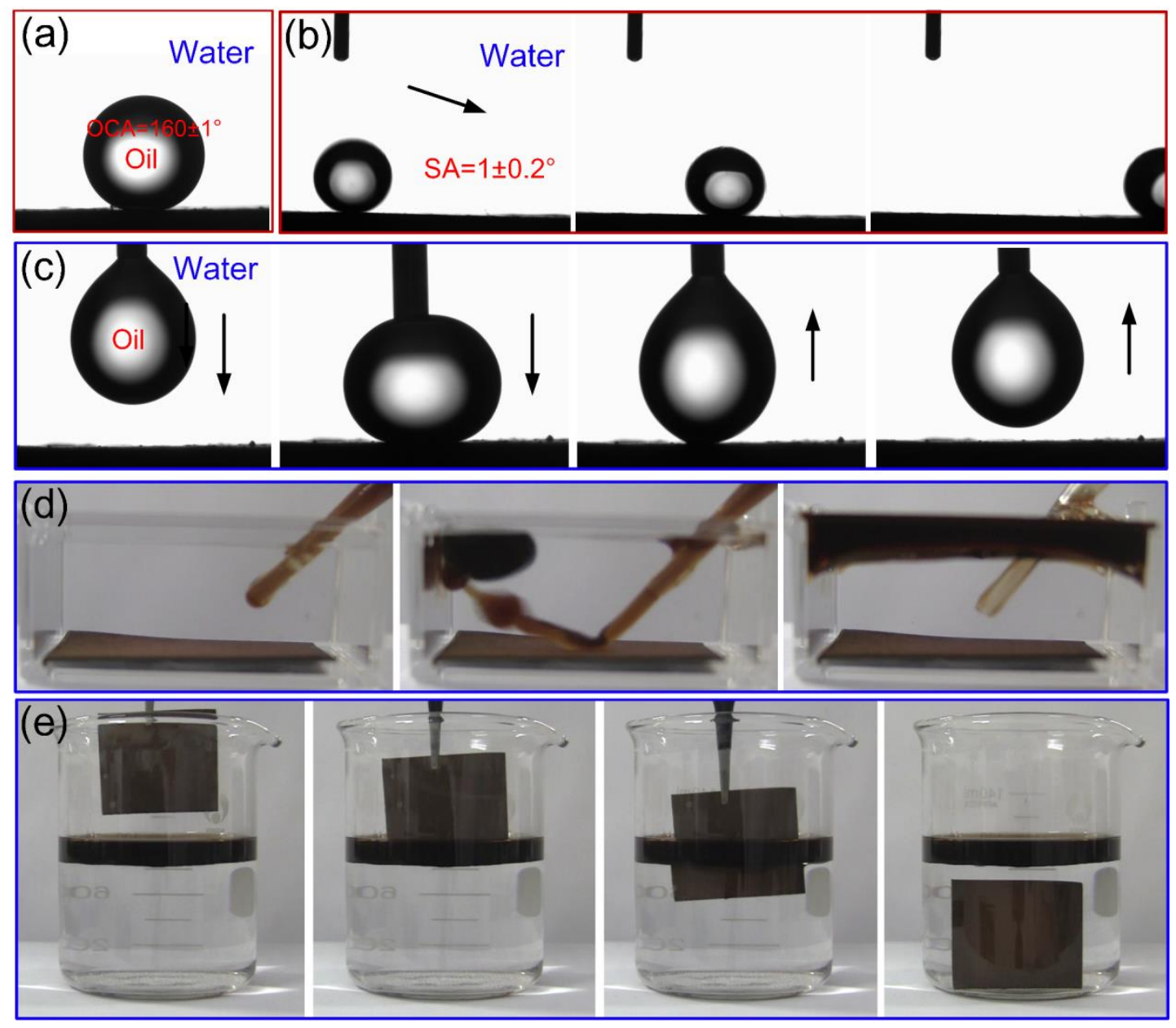

Figure 10. (a) Underwater oil contact angle and (b) sliding angle of as-prepared 
metal felt. (c) The approach, contacting and detaching process between an underwater

oil droplet and as-prepared metal felt. (d) A jet of crude oil bouncing off the asprepared metal felt immersed in water. (e) The as-prepared metal felt was dipped into crude/water mixture.

\section{CONCLUSION}

In summary, the wettability of silicon dioxide in oil environment was experimentally observed and underlying mechanism was revealed by performing MD simulations. Further, we have developed a novel, simple and low-cost technique to fabricate an underoil superhydrophilic surface by using $\mathrm{SiO}_{2} \mathrm{NPs}$ as building blocks. The as-prepared underoil superhydrophilic coating is closed-packed and ultrathin (the thickness is approximate hundreds of nanometers). This strategy enables coatings on complex substrates. The as-prepared metal felt could adsorb water droplets from oil, which endowed it the ability to separate water-in-oil emulsions. The as-prepared metal felt could separate both surfactant-free and surfactant-stabilized water-in-oil emulsions with high separation efficiency and flux though its pore size is larger than that of emulsified droplet. Its separation performance is better than most of other emulsion separation materials that are based on a size-sieving mechanism. Moreover, the asprepared metal felt retained outstanding separation performance after even 30 cycles of use, which demonstrated its excellent reusability and durability. Additionally, the distinctive wettability of underoil superhydrophilicity endowed it superior anti-fouling property. This study not only provides a new perspective on separating water-in-oil 
emulsion, but also a universal approach to develop special wettability materials.

\section{Associated content}

\section{Supporting information}

The supporting information is available free of charge on the ACS Publications website or from the corresponding author.

Supplementary figures and tables (PDF)

Movie S1 (MP4)

Movie S2 (MP4)

Movie S3 (MP4)

Movie S4 (MP4)

Movie S5 (MP4)

Movie S6 (MP4)

\section{AUTHOR INFORMATION}

\section{Corresponding author}

*(Jiadao Wang) E-mail: jdwang@mail.tsinghua.edu.cn. Telephone: +86-010-62796458.

\section{NOTES}

The authors declare no competing financial interest. 


\section{ACKNOWLEDGMENTS}

We thank the financial support from National Natural Science Foundation (grant nos. 51775296, 51375253, 51703116) and State Key Laboratory of Tribology (grant no. SKLT2018C06). Ivan P. Parkin thanks EPSRC for grant No EP/L015862/1.

\section{REFERENCES}

1 Song, P.; Cui, J.; Di, J.; Liu, D.; Xu, M.; Tang, B.; Zeng, Q.; Xiong, J.; Wang, C.; He, Q.; Kang, L.; Zhou, J.; Duan, R.; Chen, B.; Guo, S.; Liu, F.; Shen, J.; Liu, Z. Carbon Microtube Aerogel Derived from Kapok Fiber: An Efficient and Recyclable Sorbent for Oils and Organic Solvents. ACS Nano 2020, 14 (1), 595602.

2 Sun, Y.; Guo, Z. Recent Advances of Bioinspired Functional Materials with Specific Wettability: from Nature and Beyond Nature. Nanoscale Horiz, 2019, 4, $52-76$.

3 Li, K.; Ju, J.; Xue, Z.; Ma, J.; Feng, L.; Gao, S.; Jiang, L. Structured Cone Arrays for Continuous and Effective Collection of Micron-Sized Oil Droplets from Water. Nature Commun, 2013, 4, 2276.

4 Zeng, X.; Long, Q.; Yuan, X.; Zhou, C.; Li, Z.; Jiang, C.; Xu, S.; Wang, S.; Pi, P.; Wen, X. Inspired By Stenocara Beetles: from Water Collection to High-Efficiency Water-in-Oil Emulsion Separation. ACS Nano, 2017, 11, 760-769.

5 Chen, C.; Weng, D.; Mahmood, A.; Chen, S.; Wang, J. Separation Mechanism and Construction of Surfaces with Special Wettability for Oil/Water Separation. ACS 
Appl. Mater. Interfaces, 2019, 11, 11006-11027.

6 Long, M.; Peng, S.; Deng, W.; Miao, X.; Wen, N.; Zhou, Q.; Deng, W. Highly Efficient Separation of Surfactant Stabilized Water-in-Oil Emulsion based on Surface Energy Gradient and Flame Retardancy. J. Colloid Interface Sci., 2018, $520,1-10$.

7 Gupta, P.; Kandasubramanian, B. Directional Fluid Gating by Janus Membranes with Heterogeneous Wetting Properties for Selective Oil-Water Separation. ACS Appl. Mater. Interfaces, 2017, 9, 19102-19113.

8 Wu, S.; Yang, H.; Xiong, G.; Tian, Y.; Gong, B.; Luo, T.; Fisher, T. S.; Yan, J.; Cen, K.; Bo, Z.; Ostrikov, K. K. Spill-SOS: Self-Pumping Siphon-Capillary Oil Recovery. ACS Nano 2019, 13 (11), 13027-13036.

9 Ye, H.; Chen, D.; Li, N.; Xu, Q.; Li, H.; He, J.; Lu, J. Durable and Robust SelfHealing Superhydrophobic Co-PDMS@ZIF-8-Coated MWCNT Films for Extremely Efficient Emulsion Separation. ACS Appl. Mater. Interfaces, 2019, 11, $38313-38320$

10 Zhang, W.; Liu, N.; Cao, Y.; Lin, X.; Liu, Y.; Feng, L. Superwetting Porous Materials for Wastewater Treatment: from Immiscible Oil/Water Mixture to Emulsion Separation. Adv. Mater. Interfaces, 2017, 4, 1700029.

11 Wei, Y.; Qi, H.; Gong, X.; Zhao, S. Specially Wettable Membranes for Oil-Water Separation. Adv. Mater. Interfaces, 2018, 5, 1800576.

12 Zhang, W.; Shi, Z.; Zhang, F.; Liu, X.; Jin, J.; Jiang, L. Superhydrophobic and Superoleophilic PVDF Membranes for Effective Separation of Water-in-Oil 
Emulsions with High Flux. Adv. Mater., 2013, 25, 2071-2076.

13 Li, F.; Bhushan, B.; Pan, Y.; Zhao, X. Bioinspired Superoleophobic/Superhydrophilic Functionalized Cotton for Efficient Separation of Immiscible Oil-Water Mixtures and Oil-Water Emulsions. J. Colloid Interface Sci., 2019, 548, 123-130.

14 Cai, Y.; Chen, D.; Li, N.; Xu, Q.; Li, H.; He, J.; Lu, J. Superhydrophobic MetalOrganic Framework Membrane with Self-Repairing for High-Efficiency Oil/Water Emulsion Separation. ACS Sustainable Chem. Eng., 2019, 7, 2709-2717.

15 Ge, J. L.; Zong, D. D.; Jin, Q.; Yu, J. Y.; Ding, B. Biomimetic and Superwettable Nanofibrous Skins for Highly Efficient Separation of Oil-in-Water Emulsions. $A d v$. Funct. Mater., 2018, 28, 1705051.

16 Yang, C.; Han, N.; Han, C.; Wang, M.; Zhang, W.; Wang, W.; Zhang, Z.; Li, W.; Zhang, X. Design of a Janus F-TiO ${ }_{2} @$ PPS Porous Membrane with Asymmetric Wettability for Switchable Oil/Water Separation. ACS Appl. Mater. Interfaces., 2019, 11, 22408-22418.

17 Zhang, Q.; Liu, N.; Wei, Y.; Feng, L. Facile Fabrication of Hydrogel Coated Membrane for Controllable and Selective Oil-in-Water Emulsion Separation. Soft Matter., 2018, 14, 2649-2654.

18 Chen, C.; Chen, L.; Chen, S.; Yu, Y.; Weng, D.; Mahmood, A.; Wang, G.; Wang, J. Preparation of Underwater Superoleophobic Membranes via TiO2 Electrostatic Self-assembly for Separation of Stratified Oil/water Mixtures and Emulsions. $J$. Membr. Sci. 2020. DOI: 10.1016/j.memsci.2020.117976. 
19 Huang, A.; Chen, L.-H.; Kan, C.-C.; Hsu, T.-Y.; Wu, S.-E.; Jana, K. K.; Tung, K.L. Fabrication of Zinc Oxide Nanostructure Coated Membranes for Efficient Oil/Water Separation. J. Membr. Sci., 2018, 566, 249-257.

20 Luo, Z.-Y.; Lyu, S.-S.; Wang, Y.-Q.; Mo, D.-C. Fluorine-Induced Superhydrophilic Ti Foam with Surface Nanocavities for Effective Oil-in-Water Emulsion Separation. Ind. Eng. Chem. Res., 2017, 56, 699-707.

21 Zhu, Y.; Xie, W.; Zhang, F.; Xing, T.; Jin, J. Superhydrophilic In-Situ-Cross-Linked Zwitterionic Polyelectrolyte/PVDF-Blend Membrane for Highly Efficient Oi1/Wat·er Emulsion Separation. ACS Appl. Mater. Interfaces., 2017, 9, 9603-9613.

22 Yang, C.; Han, N.; Wang, W.; Zhang, W.; Han, C.; Cui, Z.; Zhang, X. Fabrication of a PPS Microporous Membrane for Efficient Water-in-Oil Emulsion Separation. Langmuir 2018, 34, 10580-10590.

23 Wen, G.; Guo, Z. Nonflammable Superhydrophobic Paper with Biomimetic Layered Structure Exhibiting Boiling-Water Resistance and Repairable Properties for Emulsion Separation. J. Mater. Chem. A., 2018, 6, 7042-7052.

24 Liu, L.; Yuan, W. A Hierarchical Functionalized Biodegradable PLA Electrospun Nanofibrous Membrane with Superhydrophobicity and Antibacterial Properties for Oil/Water Separation. New J. Chem., 2018, 42, 17615-17624.

25 Li, X.; Shan, H.; Cao, M.; Li, B. Mussel-Inspired Modification of PTFE Membranes in a Miscible THF-Tris Buffer Mixture for Oil-in-Water Emulsions Separation. J. Membr. Science., 2018, 555, 237-249.

26 Yuan, X.; Li, W.; Liu, H.; Han, N.; Zhang, X. A Novel PVDF/Graphene Composite 
Membrane Based on Electrospun Nanofibrous Film for Oil/Water Emulsion Separation. Composites Communications., 2016, 2, 5-8.

27 Yang, J.; Li, H.-N.; Chen, Z.-X.; He, A.; Zhong, Q.-Z.; Xu, Z.-K. Janus Membranes With Controllable Asymmetric Configurations for Highly Efficient Separation of Oil-in-Water Emulsions. J. Mater. Chem. A., 2019, 7, 7907-7917.

28 An, Y.-P.; Yang, J.; Yang, H.-C.; Wu, M.-B.; Xu, Z.-K. Janus Membranes with Charged CNT Coatings for Deemulsification and Separation of Oil-in-Water Emulsions. ACS Appl. Mater. Interfaces., 2018, 10, 9832-9840.

29 Hu, D.; Li, L.; Li, Y.; Yang, C. Restructuring the Surface of Polyurethane Resin Enforced Filter Media to Separate Surfactant Stabilized Oil-in-Water Emulsions via Coalescence. Sep. Purif. Tech., 2017, 172, 59-67.

30 Zhang, W.; Zhu, Y.; Liu, X.; Wang, D.; Li, J.; Jiang, L.; Jin, J. Salt-Induced Fabrication of Superhydrophilic and Underwater Superoleophobic PAA-g-PVDF Membranes for Effective Separation of Oil-in-Water Emulsions. Angew. Chem. Int. Ed., 2014, 53, 856-860.

31 Z. Shi, W. Zhang, F. Zhang, X. Liu, D. Wang, J. Jin and L. Jiang. Ultrafast Separation of Emulsified Oil/Water Mixtures by Ultrathin Free-Standing SingleWalled Carbon Nanotube Network Films. Adv. Mater., 2013, 25, 2422-2427.

32 Li, J.; Xu, C.; Guo, C.; Tian, H.; Zha, F.; Guo, L. Underoil Superhydrophilic Desert Sand Layer for Efficient Gravity-Directed Water-In-Oil Emulsions Separation With High flux. J. Mater. Chem. A., 2017, 6, 223-230.

33 Liu, M.; Lu, T.; Li, J.; Hou, Y.; Guo, Z. Underoil Superhydrophilic Surfaces: Water 
Adsorption in Metal-Organic Frameworks. J. Mater. Chem. A., 2017, 6, 1692-2699.

34 Kang, H.; Liu, Y.; Lai, H.; Yu, X.; Cheng, Z.; Jiang, L. Under-Oil Switchable

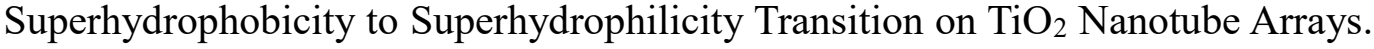
ACS Nano, 2018, 12, 1074-1082.

35 Lopes, P. E.; Murashov, V.; Tazi, M.; Demchuk, E.; MacKerell, A. D. Development of An Empirical Force Field for Silica. Application to the Quartz-Water Interface. J. Phys. Chem. B., 2006, 110, 2782-2792.

36 Berendsen, H. J. C.; Grigera, J. R.; Straatsma, T. P. The Missing Term in Effective Pair Potentials. J. Phys. Chem., 1987, 91, 6269-6271.

37 Moultos, O. A.; Tsimpanogiannis, I. N.; Panagiotopoulos, A. Z.; Trusler, J. M.; Economou, I. G. Atomistic Molecular Dynamics Simulations Of Carbon Dioxide Diffusivity in N-Hexane, N-Decane, N-Hexadecane, Cyclohexane, and Squalane. J. Phys. Chem. B., 2016, 120, 12890-12900.

38 Plimpton, S. Fast Parallel Algorithms for Short-Range Molecular Dynamics. $J$. Comput. Phys., 1995, 117, 1-19.

39 Wang, Y.; Zhu, Y.; Yang, C.; Liu, J.; Jiang, W.; Liang, B. Facile Two-Step Strategy for the Construction of a Mechanically Stable Three-Dimensional Superhydrophobic Structure for Continuous Oil-Water Separation. ACS Appl. Mater. Interfaces., 2018, 10 (28), 24149-24156.

40 Gondal, M. A.; Sadullah, M. S.; Dastageer, M. A.; Mckinley, G. H.; Panchanathan, D.; Varanasi, K. K. Study of factors governing oil-water separation process using TiO2 films prepared by spray deposition of NP dispersions. ACS Appl. Mater. 
Interfaces., 2014, 6 (16), 13422-13429.

41 Xi, B.; Verma, L. K.; Li, J.; Bhatia, C. S.; Danner, A. J.; Yang, H.; Zeng, H. C. TiO2 thin films prepared via adsorptive self-assembly for self-cleaning applications. ACS Appl. Mater. Interfaces., 2012, 4 (2), 1093-1102.

42 Peng, X.; Jin, J.; Nakamura, Y.; Ohno, T.; Ichinose, I. Ultrafast Permeation of Water through Protein-Based Membranes. Nat. Nanotech., 2009, 4, 353.

43 Lin, X.; Heo, J.; Jeong, H.; Choi, M.; Chang, M.; Hong, J. Robust Superhydrophobic Carbon Nanofiber Network Inlay-Gated Mesh for Water-in-Oil Emulsion Separation with High Flux. J. Mater. Chem. A., 2016, 4, 17970-17980.

44 Zhang, W.; Liu, N.; Cao, Y.; Chen, Y.; Xu, L.; Lin, X.; Feng, L. A Solvothermal Route Decorated on Different Substrates: Controllable Separation of an Oil/Water Mixture to a Stabilized Nanoscale Emulsion. Adv. Mater., 2015, 27, 7349-7355.

45 Ye, H.; Chen, D.; Li, N.; Xu, Q.; Li, H.; He, J.; Lu, J. Durable and Robust SelfHealing Superhydrophobic Co-PDMS@ZIF-8-Coated MWCNT Films for Extremely Efficient Emulsion Separation. ACS Appl. Mater. Interfaces., 2019, 11, 38313-38320.

46 Sun, Y.; Yang, Z.; Li, L.; Wang, Z.; Sun, Q. Facile Preparation of Isotactic Polypropylene Microporous Membranes with Bioinspired Hierarchical Morphology for Nano-Scale Water-in-Oil Emulsion Separation. J. Membr. Sci., 2019, 581, 224-235. 
Graphic abstract:
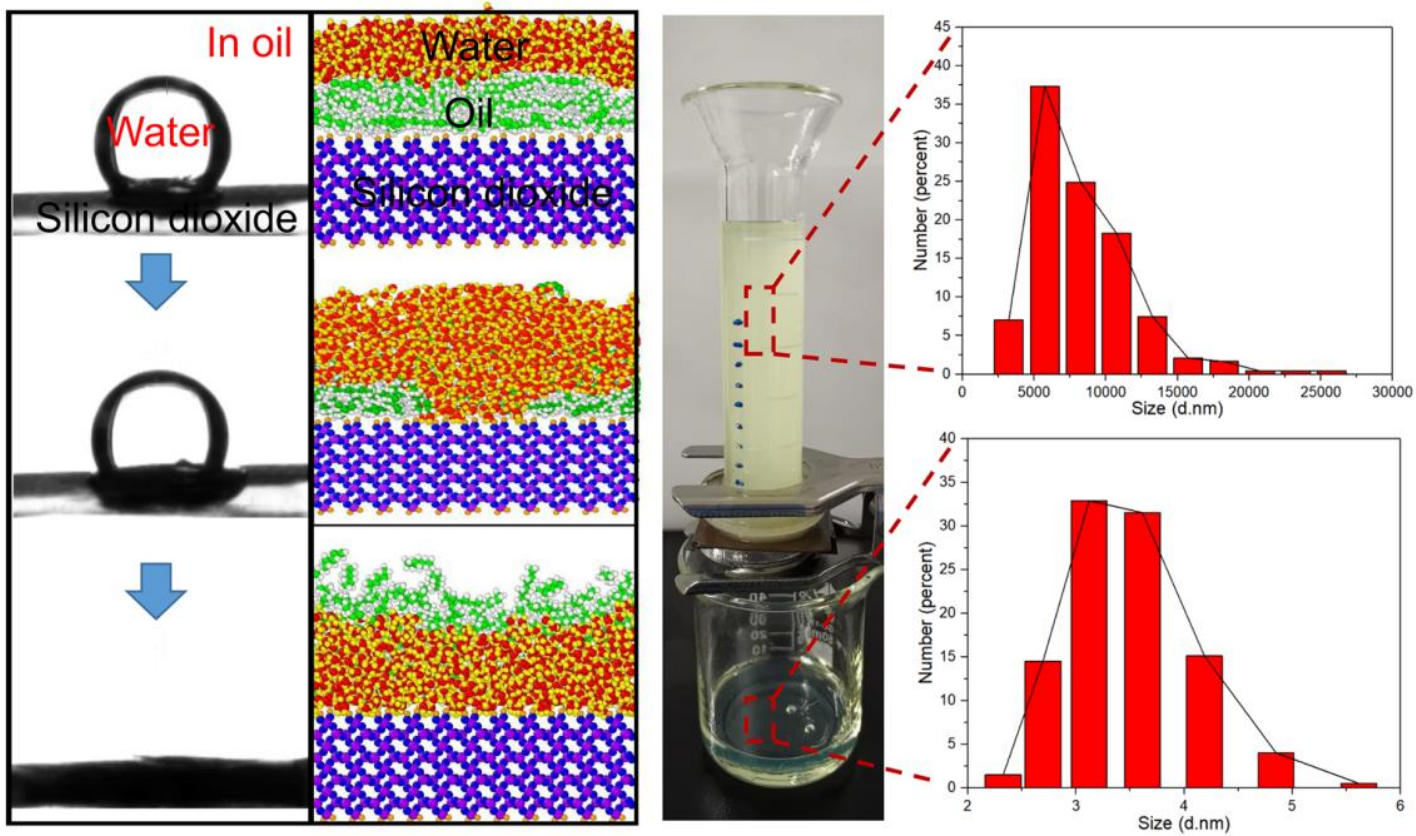